\title{
Queratoplastia Lamelar Anterior Profunda (DALK Pachy bubble) guiada por OCT transoperatorio. Caso clínico
}

\section{Deep Anterior Lamellar Keratoplasty (DALK Pachy bubble) OCT guided. Case Report}

\author{
Jorge Domene-Hinojosa ${ }^{1 *}$, Sarelle Ceja-Meraz², Ana Garcia-Treviño ${ }^{3}$, Guadalupe Espinosa-Velez², Diego \\ Garcia-Pisanty², Eduardo Cornu-Melgoza ${ }^{3}$ y Jorge Domene-Hickman ${ }^{4}$ \\ ${ }^{1}$ Director de Vision Maker; ${ }^{2}$ Córnea y Segmento Anterior, Vision Maker; ${ }^{3}$ Fellow Córnea y Segmento Anterior, IMSS UMAE 25; ${ }^{4}$ Estudiante de \\ Medicina, Instituto Tecnológico y de Estudios Superiores de Monterrey, Campus Monterrey. Monterrey, Nuevo León., México
}

\section{Resumen}

Introducción: Utilizando nueva tecnología, el microscopio OPMI Lumera ${ }^{\circledR} 700$ y RESCAN $^{T M} 700$ de la casa Zeiss, este microscopio incluye un sistema integrado de tomografía de coherencia óptica (OCT), con el cual se optimiza el procedimiento de queratoplastia lamelar anterior profunda con paquimetria transoperatoria (DALK pachy bubble) al poder observar en la pantalla la profundidad corneal durante los diferentes pasos de la cirugía, logrando mayor seguridad, y disminuyendo el riesgo de fracaso en la separación de la interfase estroma-Descemet. Teniendo en cuenta la dificultad para encontrar el plano corneal correcto, esta tecnología es un avance importante en los trasplantes lamelares. Se presenta el caso y las imágenes clínicas transoperatorias de un paciente con diagnóstico de queratocono. Caso clínico: Varón de 28 años de edad, diagnosticado hace 5 años con queratocono del ojo izquierdo, y comprobado por Pentacam ${ }^{\circ}$. Sin antecedentes de importancia, y sin enfermedad ocular o sistémica agregada. Agudeza visual del ojo derecho (OD): 20/50 sc, AVMC: 20/20 y ojo izquierdo (OI): 20/400 sc, AVMC: 20/80. A la exploración oftalmológica del Ol presenta: signo de Munson, anillo de Fleischer y estrías de Vogt, sin opacidades corneales. El paciente es intervenido quirúrgicamente con la técnica DALK pachy bubble, guiada por OCT transoperatorio en forma exitosa. Conclusión: Esta tecnología es un avance importante, que mejora la visualización transoperatoria de las capas corneales, permitiendo optimizar los pasos quirúrgicos necesarios para lograr la separación aérea de la interfase estroma-Descemet mediante la técnica de gran burbuja y paquimetria transoperatoria (DALK pachy bubble).

Palabras clave: Queratoplastia lamelar anterior profunda. Paquimetría transoperatoria. Queratocono. Tomografía de coherencia óptica. Interfase estroma Descemet.

\section{Abstract}

Introduction: Using new technology, the microscope OPMI Lumera ${ }^{\oplus}$ and RESCAN ${ }^{T M} 700$ of Zeiss company, that includes an integrated optical coherence tomography system, with which deep anterior lamellar keratoplasty with intraoperative pachymetry (DALK pachy bubble) is optimized because of the capacity to observe on the screen the corneal depth during various

Correspondencia:

*Jorge Luis Domene-Hinojosa

Av. Hidalgo 2425, consultorio 800

Col. Obispado

Fecha de recepción: 12-05-2016

Fecha de aceptación: 04-07-2016

DOI: 10.1016/j.mexoft.2016.07.004
Disponible en internet: 11-08-2016 Rev Mex Oftalmol. 2018;92(5):265-268 www.rmo.com.mx 
steps of the surgery achieving greater security and reducing the risk of failure in the separation of stromal-Descemet interface. Given the difficulty of finding the correct corneal plane, this technology is a major breakthrough in the lamellar transplants. The case and intraoperative clinical photographs in a patient diagnosed with keratoconus occurs. Case Report: Male 28 years old, diagnosed 5 years ago with keratoconus in his left eye checked by Pentacam ${ }^{\oplus}$. No history of importance or systemic pathology added. Visual acuity OD: 20/50, BCVA OD: 20/20; OI: 20/400, BCVA OI: 20/400. The ophthalmic examination left eye presents: Munson sign, Fleischer ring and Vogt striae, without corneal opacities. The patient underwent surgery with bubble DALK pachy guided intraoperative OCT successfully. Conclusion: This is a breakthrough technology that enhances intraoperative visualization of the corneal layer, thus optimizing the surgical steps needed to achieve air separation of stromal-Descemet interface by big bubble technique and intraoperative pachymetry (DALK pachy bubble).

Key words: Deep anterior lamellar keratoplasty. Intraoperative pachymetry. Keratoconus. Tomography optical coherence. Descemet stromal interface.

\section{Introducción}

Utilizando nueva tecnología OPMI Lumera ${ }^{\circledR} 700$ y RESCAN $^{\mathrm{TM}} 700$ de la casa Zeiss, este microscopio incluye un sistema integrado de tomografía de coherencia óptica (OCT), con el que se optimiza el procedimiento de queratoplastia lamelar anterior profunda con paquimetría transoperatoria (DALK pachy bubble) al poder observar por medio de la OCT imágenes de alta resolución, que nos permiten observar la microestructura corneal, incluyendo la membrana de Descemet durante los diferentes pasos de la cirugía, logrando mayor seguridad, y disminuyendo el riesgo de fracaso en la separación de la interfase estroma-Descemet. Teniendo en cuenta la dificultad para encontrar el plano corneal correcto, esta tecnología es un avance importante en los trasplantes lamelares. Se presenta el caso y las imágenes clínicas transoperatorias en un paciente con diagnóstico de queratocono.

\section{Caso clínico}

Varón de 28 años de edad, diagnosticado hace 5 años con queratocono en el ojo izquierdo (OI), comprobado por Pentacam ${ }^{\circledR}$, en donde se observa un grosor central de $378 \mu \mathrm{m}$, y en su punto más delgado de 366 ccc, ápex corneal con desplazamiento nasal inferior, queratometría media $(\mathrm{Km}) 58.6 \mathrm{D}, \mathrm{I} / \mathrm{S}$ : $2.8 \mathrm{con}$ índice de KISA elevado, con una clasificación grado 3-4 de queratocono (Figs. 1 y 2). Sin antecedentes de importancia, y sin enfermedad ocular o sistémica agregada. Agudeza visual del ojo derecho (OD): 20/50 sc, AVMC: 20/20 y del OI: 20/400 sc, AVMC: 20/80, a la exploración biomicroscópica del Ol se encuentra el signo de Munson, el anillo de Fleischer y las líneas de Vogt, sin presencia de opacidades corneales, el resto del segmento anterior sin alteraciones. El paciente es intervenido quirúrgicamente con la técnica
DALK pachy bubble, guiada por OCT transoperatorio, de forma exitosa. Los puntos clave durante la cirugía con esta tecnología son más fáciles de visualizar, como la trepanación parcial del estroma corneal, la colocación de aire intraestromal con visualización de Descemet separada del estroma corneal (Fig. 1), creándose interfase estroma-Descemet, otro punto clave es la incisión en la córnea con cuchillete hasta interfase estroma-Descemet, con visualización de la membrana de Descemet replegándose hacia el estroma (Fig. 2), momento en el cual es necesario retirar el cuchillete para evitar perforaciones inadvertidas, se muestran también las imágenes de la membrana de Descemet con un cuadrante de estroma residual (Fig. 3A), posteriormente se coloca el botón corneal donado, al cual previamente se le retiró la membrana de Descemet (Fig. 3B), para finalmente ser suturado con puntos simples de nylon 10-0, $\sin$ complicaciones.

\section{Discusión}

Desde su inicio en los años 50's, José Barraquer y colegas en Colombia disecaban 2 tercios del estroma corneal, tanto en el donador como en el receptor ${ }^{1}$, técnica que no obtuvo gran popularidad debido a la pobre agudeza visual final debido a las irregularidades en la superficie y cicatrización de la interfase. Desde 1984, que Eduardo Arenas Archila acuñó el término de queratoplastia lamelar anterior profunda, utilizando aire intraestromal para la separación de tejidos ${ }^{2}$, los trasplantes lamelares anteriores han tenido un sostenido desarrollo, evolucionando a técnicas que pueden lograr la separación de la interfase estroma-Descemet de manera segura, logrando la conservación integral de esta última, evitando restos estromales, ya que se ha comprobado que este paso es de vital importancia para lograr una agudeza visual de alta calidad. 


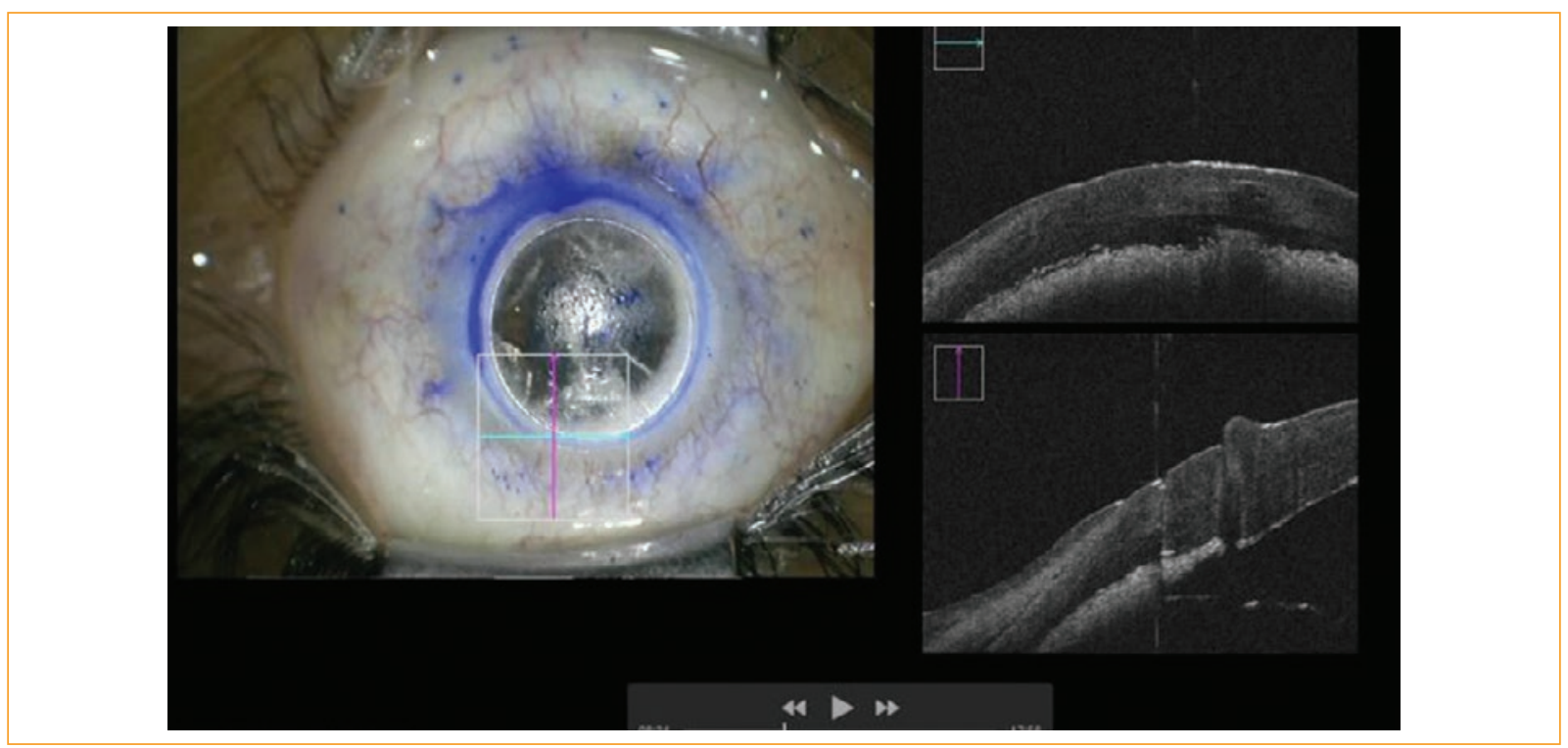

Figura 1. Colocación de aire intraestromal con visualización de Descemet separada del estroma corneal (flecha azul), creándose interfase estroma-Descemet.

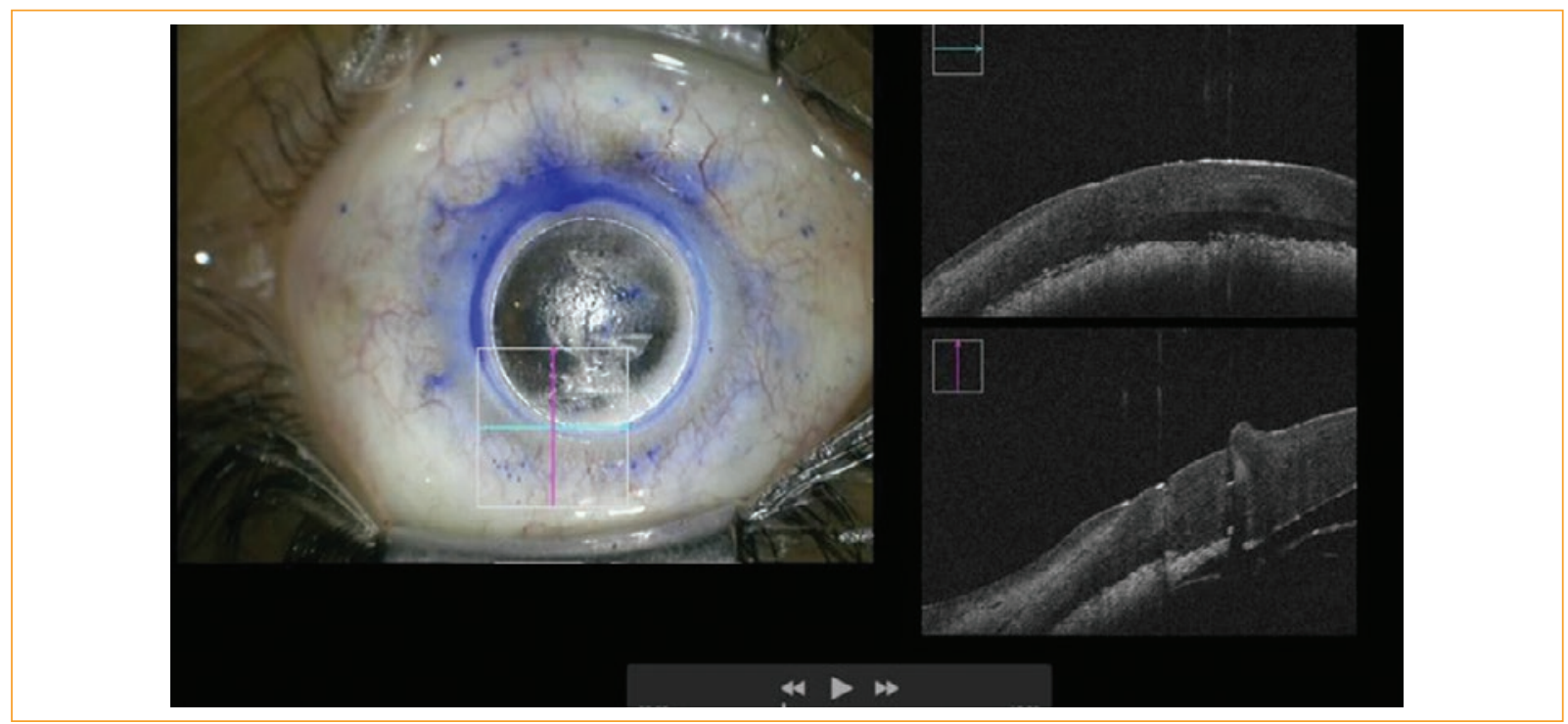

Figura 2. Incisión en cornea con cuchillete hasta interfase estroma-Descemet, con visualización de la membrana de Descemet replegándose hacia el estroma.

A través del tiempo se ha demostrado que los trasplantes lamelares anteriores profundos tienen grandes ventajas al compararlos con la queratoplastia penetrante, debido a que se disminuye el riesgo transoperatorio al evitar la trepanación corneal completa, se conserva el endotelio del receptor preservando mayor número de células $^{3}$, y evitando el rechazo inmunológico de las mismas $^{3,4}$. Otras ventajas serían, mayor resistencia al trauma, disminución en el uso de esteroides transoperatorios, menor riesgo de complicaciones como sinequias, catarata, infecciones intraoculares, glaucoma ${ }^{2,5}$ y hemorragia expulsiva, por nombrar algunas. Sin embargo, en muchos países incluyendo México esta técnica no se ha popularizado debido a la dificultad técnica y su prolongada curva de aprendizaje. Actualmente el riesgo de perforación es del 10 al 30\% en manos expertas ${ }^{4}$. 


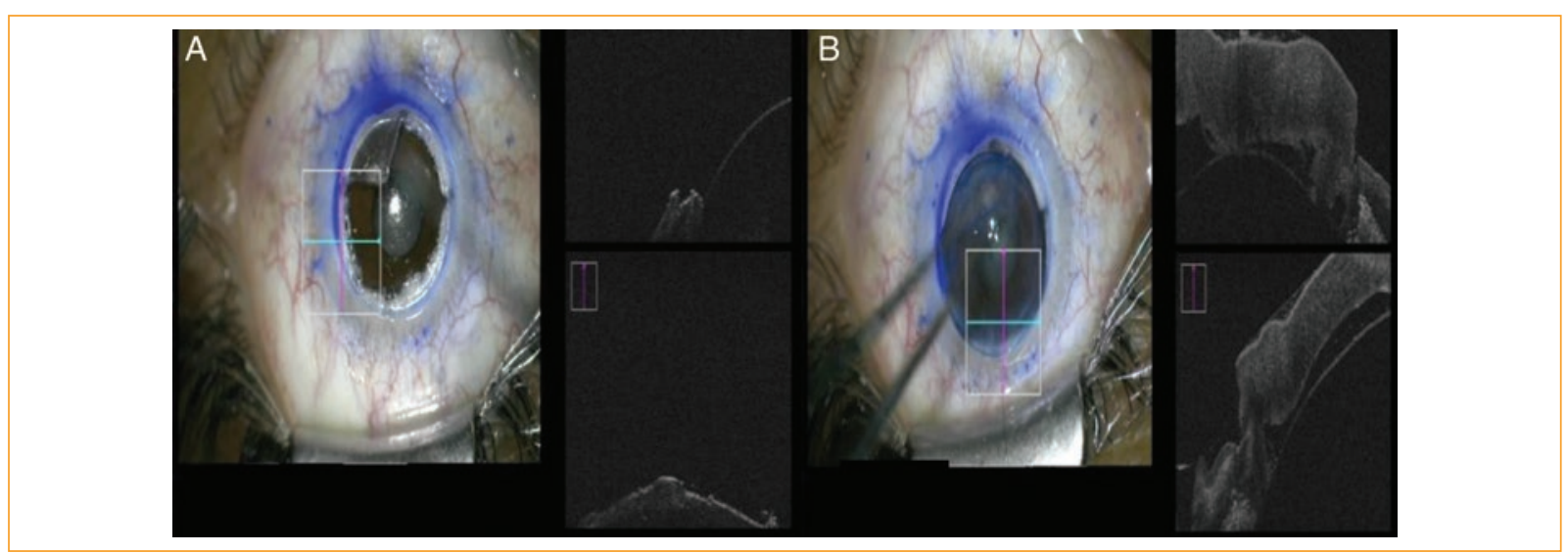

Figura 3. A: Membrana de Descemet durante su disección. B: Colocación de botón corneal donado, previa retirada de la membrana de Descemet.

\section{Conclusión}

El uso de esta nueva tecnología OPMI Lumera ${ }^{\circledR}$ 700 y RESCAN ${ }^{\mathrm{TM}} 700$, optimiza la identificación de los planos de las capas corneales al proporcionarnos un corte transversal mediante la OCT, pudiendo influir directamente en la disminución del riesgo de perforación de la membrana de Descemet al proporcionar la visualización directa en los pasos críticos como serían: trepanación corneal parcial, incisión con bisturí de diamante, disección lamelar profunda aérea y corte estromal profundo hacia la interfase aérea.

\section{Responsabilidades éticas}

Protección de personas y animales. Los autores declaran que para esta investigación no se han realizado experimentos en seres humanos ni en animales.

Confidencialidad de los datos. Los autores declaran que han seguido los protocolos de su centro de trabajo sobre la publicación de datos de pacientes.
Derecho a la privacidad y consentimiento informado. Los autores declaran que en este artículo no aparecen datos de pacientes.

\section{Financiamiento}

Los autores no recibieron patrocinio para llevar a cabo este artículo.

\section{Conflicto de intereses}

Los autores declaran no tener ningún conflicto de intereses.

\section{Bibliografía}

1. Anwar M, Teichmann K. Deep lamellar keratoplasty: Surgi-cal techniques for anterior lamellar keratoplasty with andwithout baring of Descemet's membrane. Cornea. 2002;21:374-83.

2. Shimmura S, Tsubota K. Deep anterior lamellar keratoplasty.Current Opinion in Ophthalmology. 2006;17:349-55.

3. Sogutlu Sari E, Kubaloglu A, Unal M, Pinero D, Bulut N, Erol MK, et al. Deep anterior lamellar keratoplasty versus penetratingkeratoplasty for macular corneal dystrophy: a randomized trial. Am J Ophthalmol. 2013:156:267-74.

4. Trimarchi F, Poppi E, Klersy C, Piacentini C. Deep lamellar kera-toplasty. Ophthalmologica. 2001;215:389-93.

5. Tao YK, LaBarbera M, Ehlers JP, Srivastava SK, Dupps WJ. Image-guided modified deep anterior lamellar keratoplasty (DALK)corneal transplant using intraoperative optical coherence tomo-graphy. Invest Ophthalmol Vis Sci. 2015;56:1966. 